\title{
DIGITALTECHNOLOGY Digital health: a neglected part of health
} \section{curricula?}

\author{
Authors: Mrudula Utukuri, ${ }^{A}$ Felecia D'Souza, ${ }^{\mathrm{B}}$ Alexander Deighton, ${ }^{\mathrm{B}}$ Elizabeth PV Le, ${ }^{\mathrm{C}}$ Benedict Osei-Boadu, \\ Nishita Gadi, ${ }^{\mathrm{D}}$ Ariana Axiaq, ${ }^{\mathrm{E}}$ Yuri YM Aung, ${ }^{\mathrm{F}}$ Bridget Agboola, ${ }^{\mathrm{G}}$ Chandini $\mathrm{P}$ Chand, ${ }^{\mathrm{H}}$ Connor Dibblin, ${ }^{\mathrm{I}}$ \\ Chandni R Patel, ${ }^{\mathrm{J}}$ Mohsin Abedi, ${ }^{\mathrm{K}}$ Johnathan Hirniak, ${ }^{\mathrm{K}} \mathrm{Ngan} \mathrm{H} \mathrm{Ta}^{\mathrm{L}}{ }^{\text {James HF Rudd }}{ }^{\mathrm{M}}$ and Rajiv Sethi ${ }^{\mathrm{N}}$
}

\begin{abstract}
With growing government investment and a thriving consumer market, digital technologies are rapidly transforming our means of healthcare delivery. These innovations offer increased diagnostic accuracy, greater accessibility and reduced costs compared with conventional equivalents. Despite these benefits, implementing digital health poses challenges. Recent surveys of healthcare professionals (HCPs) have revealed marked inequities in digital literacy across the healthcare service, hampering the use of these new technologies in clinical practice. Furthermore, a lack of appropriate training in the associated ethical considerations risks HCPs running into difficulty when it comes to patient rights. In light of this, and with a clear need for dedicated digital health education, we argue that our focus should turn to the foundation setting of any healthcare profession: the undergraduate curriculum.
\end{abstract}

KEYWORDS: education, digital health, undergraduate

DOI: $10.7861 /$ fhj.2021-0102

\section{Introduction}

Digital health, defined as the integration of technology into healthcare to tackle current and future challenges, is increasingly

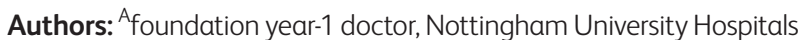
NHS Trust, Nottingham, UK; ${ }^{\mathrm{B}}$ medical student, Barts and The London School of Medicine and Dentistry, London, UK; ${ }^{C}$ MB/PhD student, University of Cambridge School of Clinical Medicine, Cambridge, UK; D medical student, Anglia Ruskin University, Chelmsford, UK; ${ }^{\text {Emedical }}$ student, Queen's University Belfast, Belfast, UK; F academic foundation year-1 doctor, Barking, Havering and Redbridge University Hospitals NHS Trust, London, UK; ${ }^{G}$ medical student, Edinburgh Medical School, Edinburgh, UK; ${ }^{H}$ medical student, Hull York Medical School, Hull, UK; Imedical student, King's College London, London, UK; ' foundation year-1 doctor, Barts Health NHS Trust, London, UK; ${ }^{\text {foundation year-1 }}$ doctor, Mid and South Essex NHS Foundation trust, Basildon, UK; Loundation year-2 doctor, East Anglian Foundation School, Norwich, UK; " honorary consultant cardiologist and deputy chief clinical information officer, Cambridge University Hospitals NHS Foundation Trust, Cambridge, UK; ${ }^{\mathrm{N}}$ specialty registrar, Manchester University NHS Foundation Trust, Manchester, UK being recognised as a key component of patient care. ${ }^{1} \mathrm{~A}$ thriving direct-to-consumer healthcare market has led to more patients engaging with these technologies and, likewise, so must healthcare professionals (HCPs). While the COVID-19 pandemic has undoubtedly driven more HCPs to actively engage with digital health, it has also highlighted the spectrum of digital health literacy that currently exists within the workforce. As technology becomes commonplace in everyday healthcare, there is a growing need for all HCPs to be able to use, interpret and discuss digital health solutions. The question then remains of when best to instil digital health literacy in the healthcare workforce. We postulate that the ideal period to develop such competencies may be undergraduate health curricula.

Although aspects of digital health have yet to make the leap to clinical practice, digital technologies are increasingly being used to deliver routine care. Global telehealth users have grown from approximately 350,000 in 2013 to an estimated 7 million just 5 years later. ${ }^{2}$ Meanwhile, the recently inaugurated NHS Genomic Medicine Service aims to carry out a record 5 million analyses over the next 5 years, and more than $96 \%$ of general practices now use electronic health record systems. ${ }^{3,4}$ In July 2020, the UK secretary of state for health and social care claimed that 'Zoom medicine' or video consultation would become the 'new normal', and undoubtedly the COVID-19 pandemic has only further emphasised the necessity of such virtual consultations. ${ }^{5}$ Simultaneously, patients are independently driving the uptake of digital health solutions through their increased use of wearables (like smartwatches and smartphones), with 3.7 billion healthcarerelated applications being downloaded in 2017.6 This bi-directional adoption of digital health, therefore, requires a future healthcare service of practitioners that are better equipped to engage with, innovate, evaluate and even prescribe digital health interventions.

\section{Current problems and attempts to progress}

Crucially, however, despite its potential benefits, digital health adoption has clear accompanying challenges. Greater automation risks dehumanising patient care, and the four pillars of medical ethics are more relevant in this digital age than ever before. To do no harm, HCPs must be sufficiently trained in digital health use, effective data management, and awareness of the accompanying risks. These risks include data and automation biases, where data and algorithms may mirror human biases in decision making, and 
HCPs may accept machine advice without clinical validation. ${ }^{1}$ Justice in resource allocation must also be considered to prevent widening the digital divide and further reinforcing existing social inequalities. Simultaneously, patient autonomy must remain a priority. Anonymised patient data is the first step towards privacy, however, patient trust will likely also depend on concrete measures of accountability and transparency regarding data management. ${ }^{7}$ Before engaging with and prescribing digital interventions, HCPs must not just be able to assess the digital literacy of their patients, including their willingness to engage and possible barriers to uptake (eg accessibility and cost), but also assess their own digital literacy and abilities to navigate the nuances of digital health interventions.

Notably, there have been clear, concerted efforts made in the UK healthcare system to adopt innovative models of digital care and better train their staff. Governmental bodies such as NHSX and NHS Digital, due to merge with NHS England, have been tasked with driving digital transformation programmes, while HCPs, benefiting from leadership opportunities (such as the Topol fellowships and Health Education England (HEE) NHS Digital Academy Digital Health Leadership programme) are expected to meet an array of new standards including HEE's digital capabilities framework and the General Medical Council's generic capabilities framework. Importantly, however, accessibility to training remains one major barrier to the universal uptake of digital health by HCPs, prompting The Topol Review to highlight the need for improved access to training and support if we are to create a digitally literate workforce. ${ }^{1}$ Consequently, despite the creation of these postgraduate digital health courses, flexible portfolio training and the introduction of formal digital health and entrepreneurship training posts in the UK, opportunities remain skewed towards those with prior qualifications, understanding or interest. ${ }^{8,9}$ Arguably, this then only serves to highlight the lack of a standardised approach to postgraduate digital health education, risking further inequitable digital health literacy in the workforce. Awareness of required competencies, and access to training and support, will thus need to be improved as a fundamental shift in the delivery of healthcare takes place over the next 2 decades.

\section{The undergraduate curriculum as a means of change}

To circumvent this issue, and develop key digital competences prior to entering clinical practice, we argue that we should look to the very foundation of each HCP's career: the undergraduate health curricula. Few medical schools, as one such example of a provider of the undergraduate health curriculum, have incorporated digital health education into their curricula, potentially missing out on a key opportunity to encourage digital health literacy from the very beginning. Key exceptions are Barts and The London School of Medicine and Dentistry, University College London Medical School and Warwick Medical School, all of which have launched digital health courses in recent years, with Warwick students in particular benefiting from the Institute of Digital Healthcare. 'Barts X Medicine', for example, involves a series of seminars from global digital health influencers, patient representatives and entrepreneurs, alongside a 2-month-long competition where students can develop their own digital health business model. By demonstrating the importance of technology in an increasingly digital healthcare service, and developing key entrepreneurial skills, the project hopes to encourage future HCPs to not only advocate for digital health but also to innovate themselves.
Rather than hope for other medical schools to follow suit, however, perhaps what would be most beneficial is to standardise a nationwide digital health curriculum for all medical schools and allied health courses. This would ensure equal baseline digital health training for all future practitioners, with further opportunities available down the line for those more inclined. Understandably, this would be no small undertaking and research into what should be included in such a curriculum would be sorely needed. The needs of students, educators and experts in the digital health field would need to be comprehensively mapped in order to determine how best to align any potential curriculum with the existing postgraduate digital capabilities framework. Similarly, the method by which such teaching is delivered and assessed must be carefully considered, for example, whether by formative or summative assessments. As of 2021, just one UK study has attempted to assess these needs, an ongoing study by the Faculty of Digital Health, highlighting the need for further work in this area. ${ }^{10}$

\section{Conclusion}

The utilisation of digital health is advancing rapidly, in part, accelerated by the current pandemic. HCPs will thus need to be competent in using and interpreting information from these technologies, as well as be duly equipped to deal with any challenges that may result. Despite this, digital health education in the UK remains highly varied, and the point at which practitioners should develop these critical skills remains unclear and under-researched. What is clear is that early development of such competencies provides a strong foundation for future clinical practice, particularly if developed as a continuous, multidisciplinary curriculum around existing NHS digital capabilities frameworks. Such a curriculum would undoubtedly provide a coherent approach across undergraduate and postgraduate education, and a better understanding of the current provisions and challenges of digital health education is essential if we are to better prepare the next generation of HCPs for the digital future.

\section{Acknowledgements}

We would like to thank Prof Shafi Ahmed (Founder, Barts X Medicine) and Dr Sandeep Bansal (Faculty, Barts X Medicine) for their valuable advice during the production of this manuscript.

\section{Conflicts of interest}

Mrudula Utukuri, Alexander Deighton, Elizabeth PV Le, Benedict Osei-Boadu, Nishita Gadi, Ariana Axiaq, Yuri YM Aung, Bridget Agboola, Chandini Parsan Chand, Chandni R Patel, Mohsin Abedi, Johnathan Hirniak, Ngan H Ta and Rajiv Sethi report involvement in The Faculty of Digital Health UK Steering Group.

Rajiv Sethi is a faculty member of Barts X Medicine. Alexander Deighton and Chandni Rajesh Patel are previous participants of the Barts X Medicine scheme and medical students at Barts and The London School of Medicine and Dentistry. Felecia D'Souza is a medical student at Barts and The London School of Medicine and Dentistry.

\section{References}

1 Health Education England. The Topol Review. HEE, 2019. https:// topol.hee.nhs.uk/the-topol-review [Accessed 09 July 2020]. 
2 Statista. Projected number of telehealth patients worldwide 2013 to 2018. Statista, 2018. www.statista.com/statistics/302641/ global-telehealth-market-patients [Accessed 09 July 2020].

3 Department of Health and Social Care. Matt Hancock announces ambition to map 5 million genomes. DHSC, 2018. www.gov.uk/ government/news/matt-hancock-announces-ambition-to-map5-million-genomes [Accessed 17 July 2020].

4 Imison C, Castle-Clarke S, Weston R, Edwards N. Delivering the benefits of digital health care. Nuffield Trust, 2016. www.nuffieldtrust. org.uk/files/2017-01/delivering-the-benefits-of-digital-technologyweb-final.pdf [Accessed 15 July 2020].

5 BBC News. 'More Zoom medicine needed' in NHS says Hancock. BBC, 2020. www.bbc.co.uk/news/amp/health-53592678 [Accessed 31 July 2020].

6 Statista. Number of mHealth app downloads worldwide from 2013 to 2017. Statista, 2017. www.statista.com/statistics/625034/ mobile-health-app-downloads [Accessed 09 July 2020].

7 Vayena E, Haeusermann T, Adjekum A, Blasimme A. Digital health: meeting the ethical and policy challenges. Swiss Med Wkly 2018:148:w14571. https://smw.ch/article/doi/smw.2018.14571 [Accessed 15 July 2020].

8 Royal College of Physicians. Flexible portfolio training. RCP, 2020. www.rcplondon.ac.uk/projects/flexible-portfolio-training [Accessed 03 October 2021].

9 UK Foundation Programme. Foundation Priority Programmes. UK Foundation Programme, 2020. www.foundationprogramme. nhs.uk/wp-content/uploads/sites/2/2019/11/Foundation-PriorityProgrammes-F [Accessed 17 July 2020].

10 Gokulakrishnan D, Butler SE, Proctor DW, Ferry ML, Sethi R. Social media: powering open access collaboration and global knowledge exchange. Med Educ 2021;55:1216-8.

Address for correspondence: Alexander Deighton, Barts and The London School of Medicine and Dentistry, Queen Mary University of London, Garrod Building, Turner Street, Whitechapel, London E1 2AD, UK.

Email: a.deighton@smd17.qmul.ac.uk

Twitter:@AlexJDeighton 